\title{
A Method for the Design of UFLS Scheme with Dynamic Correction
}

\author{
Zhaoou Song ${ }^{1}$, Junyong Liu ${ }^{1}$, Youbo Liu ${ }^{1}$, Masoud Bazargan ${ }^{2}$, Wuxing Liang ${ }^{2}$ \\ ${ }^{1}$ School of Electrical Engineering and Information, Sichuan University, Chengdu, China \\ ${ }^{2}$ LSTOM Grid Research \& Technology Centre, Stafford ST17 4LX, UK \\ Email: cklein927@163.com
}

Received March, 2013

\begin{abstract}
This paper presents an adaptive Under Frequency Load Shedding scheme based on Wide Area Measurement System. Due to the lack of enough adaptability to the operation state of the system, the traditional successive approximation under frequency load shedding method will cause excessive cut or undercut problems inevitably. This method consists first in a comprehensive weight index including load characteristics and inertias of generators. Then active-power deficit calculation based on the Low-order Frequency Response Model, concerning the effect of voltage was put forward. Finally, a dynamic correction of the load shedding amount was proposed to modify the scheme. This approach was applied to IEEE-39 system and the simulation results indicated that the proposed method was effective in reducing the load shedding amount as well as the frequency recovery time.
\end{abstract}

Keywords: UFLS; WAMS; Active- power Deficit; Comprehensive Weight

\section{Introduction}

As the last resort against system blackouts, UFLS schemes implemented today are conventional and adaptive schemes. The former one is generally based on off-line calculation, shedding a predefined amount of load in case frequency and/or the rate of change of frequency (ROCOF) fall below a certain threshold with a time delay. While the latter one allocates the active- power deficit in each load shedding step, taking online conditions into account.

Reference [1] proposes a new methodology based on risk management and quantitative analysis for the time response curves, and coordination between UFLS and UVLS is studied. A UFLS scheme, based on non-recurve Newton algorithm to estimate the frequency and the frequency change ratio, is put forward [2]. Concerning the frequency characteristic difference of different load nodes, reference [3] puts forward a load shedding scheme base on comprehensive weights. References $[4,5]$ propose a low-order model to calculate the response of the system with disturbance. A load shedding method ensuring the stability of both frequency and voltage is studied in [6]. In a word, references mentioned above are all based on the low-order frequency response model to calculate the deficit power. Nevertheless, the model ignores the effect of voltage on the deficit power, resulting in some error in the deficit power calculation.

The literatures mentioned above, irrespective of the effect of generator inertia on the grid dynamic frequency, seldom analyze the correlation properties of these two coupling parameters in UFLS. Besides, in the multimachine system, there is a close relationship between the frequency variation and generator inertia time constant. As a result, the effect of generator inertia on the frequency shall not be neglected.

This paper proposes a new adaptive UFLS based on WAMS. The main elements of the proposed WAMSbased scheme are given below.

1) A power deficit calculation based on WAMS considering voltage effect factor.

2) A comprehensive load shedding criterion construction concerning load characteristics and the effect of generators on loads.

3) A dynamic correction of load shedding amount in accordance with the system self regulation ability and real-time frequency change rate.

\section{Comprehensive Load Shedding Criterion}

Considering the differences of load frequency characteristic and generation unit inertia, calculate the comprehensive weights of each node in the process of load shedding. Based on the weights obtained to allocate the load shedding amount and determine the location.

\subsection{The Path Distribution Weight of Generator Inertia}

In multi-machine system, the frequency variation with 
active power disturbance has a close relationship with generator inertial time constant and disturbance site. Calculate the frequency change rate instantly when the disturbance occurs (3 time cycles after the disturbance), and use Equation (4) to calculate the deficit power $P_{d e-}$ $f_{i}(i=1,2, \ldots \mathrm{n})$ of each unit to measure generator disturbance degree. In under-frequency state, the larger deficit power is, the greater degree of unit is. The nearby load of unit with large deficit power should be given priority to be cut to stabilize the system as soon as possible. Choose the maximum value $P_{\text {demax }}$ as the base value to normalize all the $P_{\text {defi }}$. The disturbance degree weight then can be expressed by:

$$
\beta_{i j}=P_{\text {defi }} / P_{\text {def } \max },
$$

In which, $n$ stands for the number of generators; $m$ is the number of loads; $\beta_{i j}$ is the weight of $j$ th load which is near to $i$ th generator. As the analysis mentioned above, the larger $\beta_{i j}$ is, the greater disturbance degree of unit is. Shed these sort of loads first can facilitate the recovery of frequency.

With regard to the ownership of the load, the shortest electric distance from load node to generator is adopted to differentiate the ownership. Assume the number of node is $a$, the number of edge is $b$, calculate the adjacency according to the weighted power system network theory. Apply Floyd algorithm to the matrix WG to figure out the shortest electric distance matrix D. Calculate all the electric distance from $j$ th load node to all the generators according to matrix $\mathrm{D}$, choose the shortest distance to obtain the load weight $\beta_{i j}$.

\subsection{Load Frequency Characteristic Weight}

Load active-frequency characteristic refers to the characteristic that the load active power varies with the change of frequency:

$$
\begin{aligned}
P_{L}= & a_{0} P_{L N}+a_{1} P_{L N}\left(\frac{f}{f_{N}}\right)+a_{2} P_{L N}\left(\frac{f}{f_{N}}\right)^{2} \\
& +\ldots+a_{n} P_{L N}\left(\frac{f}{f_{N}}\right)^{n}
\end{aligned}
$$

In which, $a_{i}$ is the proportion of load which is proportional to the $i$ times of frequency; $P_{L N}$ is the rated load of system; $f_{N}$ is the rated frequency.

In real power system, the load which is proportional to the 3 times of frequency is rather fewer; Equation (12) can be simplified by Equation (13):

$$
P_{L^{*}}=a_{0}+a_{1} f *+a_{2} f *^{2}+a_{3} f *^{3}
$$

Further, we can obtain:

$$
K_{L}=\frac{d P_{L^{*}}}{d f *}=a_{1}+2 a_{2} f *+3 a_{3} f *^{2}
$$

In which, $K_{L}$ is the load frequency regulation coeffi- cient and each load corresponds to different $K_{L}$.

When the frequency drops, the load with bigger $K_{L}$ absorbs less active power from the system. Therefore, calculate the reciprocals of $K_{L 1}, K_{L 2}, K_{L 3} \ldots K_{L m}$ and choose the maximum value $\left(1 / K_{L m i n}\right)$ as the base value to normalize all the coefficients. The load static characteristic weight then can be expressed by:

$$
\lambda_{j}=\frac{K_{L \text { min }}}{K_{L j}}
$$

In which, $K_{L j}$ is the frequency regulation coefficient of $j$ th load node; $K_{L \min }$ is minimum value of $\mathrm{m}$ coefficients.

The analysis indicates that shed the load with small $K_{L}$ first can reduce the absorption of active power from system, facilitating the recovery of frequency. The greater $\lambda_{j}$ is, the $\mathrm{KL}$ is smaller, in order to make full use of load self frequency regulation ability, the load with greater $\lambda_{j}$ should be chosen as the key target of load shedding.

\subsection{The Comprehensive Weight}

Considering the load static characteristic and the disturbance degree load weight comprehensively, a weighted product of these two weights is proposed. In the process of load shedding, the real-time frequency variation of each node can be provided by WAMS, which can be utilized to calculate the load characteristic and disturbance degree, so as to figure out the comprehensive weight of each load node to act as the criterion of load shedding amount and site.

Define the comprehensive weight as:

$$
\varphi_{j}=\lambda_{j} \cdot \beta_{i j}, \forall i, j
$$

In the process of UFLS, the load with greater comprehensive weight is preferred to be assigned large shedding amount to recover the system as soon as possible.

\section{Deficit Power Calculation Base on WAMS}

\subsection{Deficit Power Calculation Based on Frequency Response Model}

In order to estimate the deficit power of whole system accurately, it is reasonable to imagine an equivalent generation unit that describes the average behavior of all the generators. This equivalent unit is called COI. The inertia constant and frequency of COI are defined respectively as follows:

$$
f_{C O I}=\frac{\sum_{i=1}^{n} H_{i} \cdot f_{i}}{\sum_{i=1}^{n} H_{i}}
$$

In which, $f_{i}$ is the frequency of $i$ th generator; $n$ is the number of generators. 


$$
H_{e q}=\frac{\sum_{i=1}^{n} H_{i} \cdot S_{i}}{\sum_{i=1}^{n} S_{i}}
$$

In which, $H_{i}$ is the inertia constant of ith generator; $\mathrm{Si}$ is the rated power of $i$ th generator.

According to the frequency variation curve the WAMS provided, the frequency variation rate of the initial disturbance moment can be calculated by numerical calculation method:

$$
\frac{d f(n)}{d t}=\frac{(f(n)-f(n-1))}{T_{i}}
$$

Then the deficit power can be obtained by:

$$
\begin{gathered}
P_{\text {def }}=\frac{2 H_{e q}}{f_{N}} \frac{d f_{C O I}}{d t} S_{e q} \\
S_{e q}=\sum_{i=1}^{n} S_{i}
\end{gathered}
$$

In which, the $f_{N}$ is the rated frequency.

\subsection{The Voltage Influence Factors of Deficit Power Calculation}

The frequency response model ignores the effect of voltage on the deficit in the initial disturbance moment. However, in the process of frequency decrease, node voltage also drops in the meantime, which reflects an existence of a coupling relationship between the active power deficit and the reactive power deficit $[7,8]$. In the $1 \sim 2$ seconds of disturbance, voltage variation acts as the leading role in the effect on the change of load active power, henceforth, the frequency replaces the voltage to play a decisive role. Due to the fact that the change of load reflects the deficit active power, at the initial disturbance moment, the voltage will change immediately; therefore, the instantaneous response of load to the voltage variation can not be ignored. The load model concerning the influence of voltage is built below:

$$
P_{L}=\sum_{j=1}^{m} P_{L 0, j}\left(\frac{U_{j}}{U_{0, j}}\right)
$$

On the basis of [9], the deficit power calculations considering the effect of load voltage mutation are given as follows:

$$
\begin{gathered}
P_{\text {def }}=P_{\text {tur }}-P_{L} \\
P_{\text {shed }}=P_{\text {tur }}-P_{L 0} \\
P_{\text {shed }}=P_{1}+P_{2} \\
P_{1}=\frac{2 H_{e q}}{f_{N}} \frac{d f_{C O I}}{d t} S_{e q} \\
P_{2}=\sum_{j=1}^{m} P_{L 0, j} \cdot\left[\left(\frac{U_{j}}{U_{0, j}}\right)^{\alpha}-1\right]
\end{gathered}
$$

In which, $P_{t u r}$ is the output of turbine; $P_{L}$ is the load; $P_{\text {shed }}$ is the deficit power concerning the voltage influence; $\mathrm{m}$ is the number of load nodes; $P_{L 0, j}$ is the active power of $j$ th load node before disturbance, $U_{0, j}$ is the voltage of $j$ th load node before disturbance, $U_{j}$ is the voltage of $j$ th load node after disturbance; $\alpha$ is the voltage influence factor and $\alpha=1$ is chosen in this paper. The calculation result of Equation (9) is chosen as the base value of load shedding amount.

\section{UFLS Scheme with Dynamic Correction}

On the basis of the measurement data provided by WAMS, calculate the deficit power using Equation (9) as the base UFLS shedding amount. With reference to the real UFLS, the conditions for activation of individual steps are equal to the following threshold frequencies: $49.2 \mathrm{~Hz}, 49 \mathrm{~Hz}, 48.8 \mathrm{~Hz}$. All of the four predefined distributions $P_{\text {shed, }}(k=1,2,3)$ are set in accordance with the traditional UFLS scheme: $30 \%, 30 \%, 40 \%$ of the deficit power.

Due to the effect of speed governor and load frequency characteristic, after each load shedding step implemented, the frequency change rate does not remain constant, which will lead to the change of deficit power. Prior to the implementation of load shedding, the frequency decreases dramatically, i.e. the frequency drop rate is the largest. After each load shedding step, the frequency is relieved, which in return reduces the deficit power, reducing the corresponding load shedding amount. However, the traditional scheme lacks of the correction of load shedding, which will inevitably result in overcut, so it's necessary to adjust the predefined load shedding steps to the primary frequency-control reaction. It is obvious from Equation(4) that the deficit power is a nearly linear function of frequency change rate, in general, the $x \%$ lowering of $P_{\text {def }}$ is reflected in an $x \%$ lowering of frequency change rate. Based on this linearity concept, before the $k$ th shedding step is activated, with the use of WAMS, monitor the changes in $d f_{\mathrm{COI}} / d t$ between two neighboring shedding steps and compare it to the ini$\operatorname{tial}\left(d f_{C O I}, \max / d t\right)$ value, and the percentage change of $d f_{C O I} / d t$ is calculated:

$$
\Delta k \%=\frac{d f_{C O I, k-1} / d t-d f_{C O I, k} / d t}{d f_{C O I, \max } / d t} \cdot 100
$$

Keeping in mind the presented linearity concept, the frequency variation rate change gradient $\Delta_{\mathrm{k}} \%$ demonstrates the change in deficit power. As a result, the upcoming shedding step can be altered from its predefined value $P_{\text {shed, } k}$ according to:

$$
P_{\text {shed, } k}^{\prime}=P_{\text {shed }, k}-\Delta_{k} \%
$$

After the achievement of $k$ th shedding amount, the requirement of control rapidity is taken into account to 
introduce a new method to distribute the $P^{\prime}{ }_{\text {shed, } k}$ in combination with the proposed comprehensive load weight. The shedding amount of each load node is calculated by:

$$
P_{\text {shed }, j}=P_{\text {shed }, k}^{\prime} \times \frac{\varphi_{j}}{\sum_{j \in M} \varphi_{j}}
$$

In which, $M$ is a set of all load nodes. Consequently, activate each shedding step according to the thresholds to gradually restore the frequency.

The detailed process of UFLS is shown in Figure 1.

\section{Test System Proposed UFLS Scheme}

A load shedding procedure was tested on an island of a 39-bus IEEE test system, which is given in Figure 2.

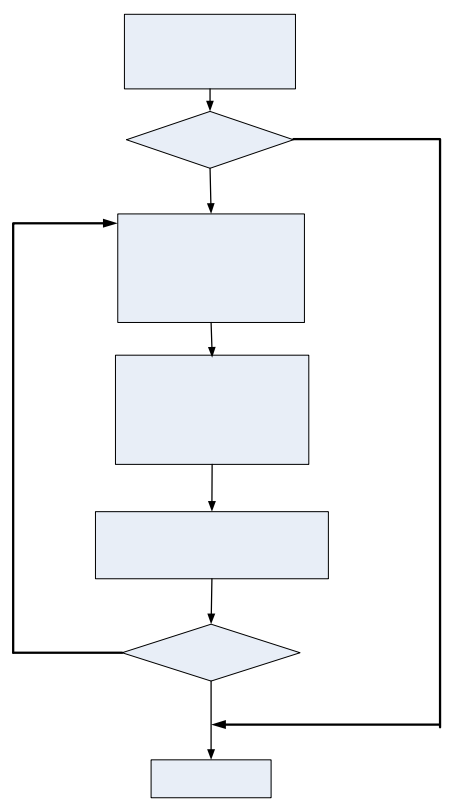

Figure 1. Flow chart of UFLS.

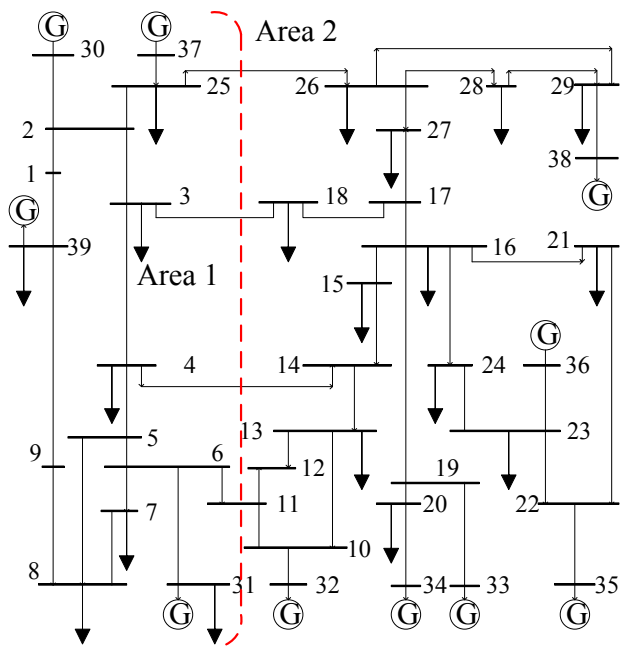

Figure 2. IEEE39 nodes power system.
At 4 seconds, line 25-26, 3-18, 4-14 and 6-11 are cut simultaneously; area 1 becomes an isolated grid with a large deficit power. Figure 3 demonstrates the variation of frequency provided by WAMS at the very moment that the grid splits.

This paper selects the data from WAMS within 3 time cycles after disturbance, using Equation (3) calculates and averages, as the system frequency variation rate of this moment. At the instant that frequency drops, calculates the deficit power of this moment using Equation (5) as the base load shedding amount. The variation of voltage while islanding is shown in Figure 4.

The combination with Figure $\mathbf{3}$ and Figure $\mathbf{4}$ indicates that the voltage of load nodes also plunges dramatically at the instant frequency declines, especially 1-2 s after disturbance. Henceforth, due to the regulation of excitation system, the voltage gradually restores while the frequency continues to decrease. It verifies that the voltage variation plays the leading role on the change of load active power at the initial stage of disturbance; the frequency replaces it as main factor afterwards. Irrespective of the influence of voltage, the deficit power is: $P_{\text {def }}$ $=5.5025$ p.u and it is $P_{\text {def }}=5.3911$ p.u when voltage is

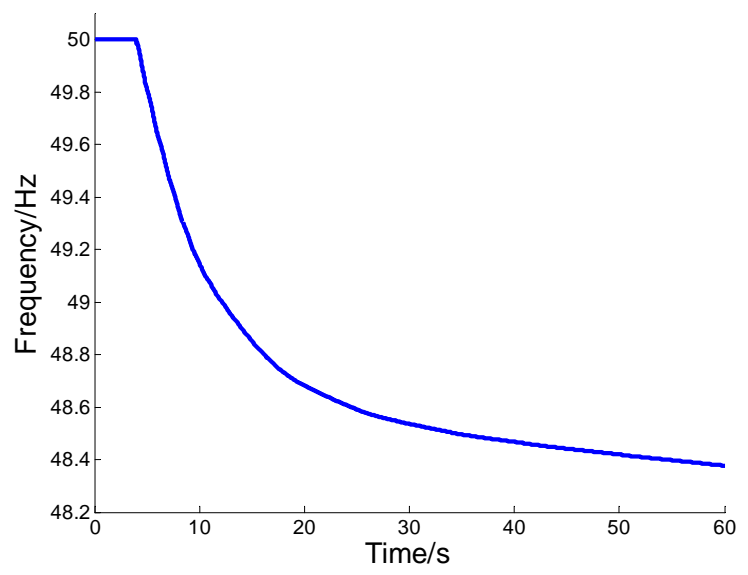

Figure 3. The frequency variation curve while islanding.

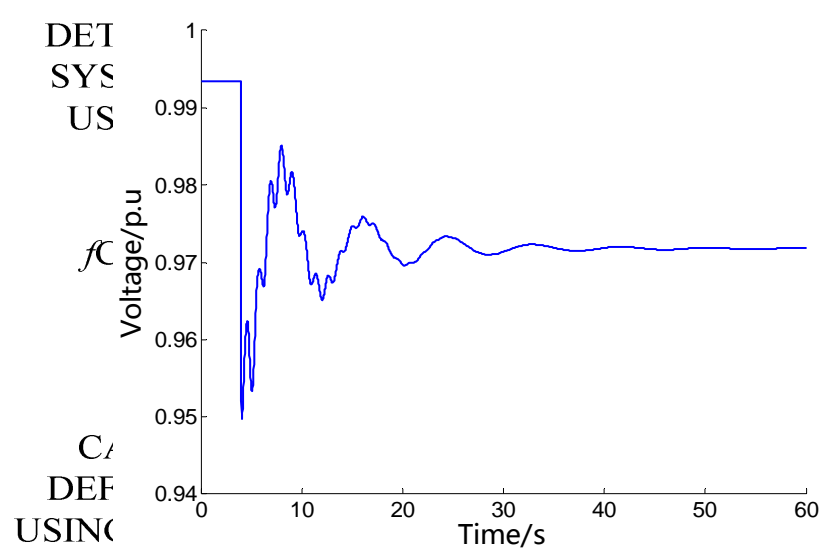

TRAJECTORY Figure 4 . The voltage variation curve while islanding. 
taken into account. It is obvious that the voltage mutation can not be ignored in the estimation of deficit power. It will cause great error or even likely lead to problems such as overcut without concerning the influence of voltage.

It is a fact that the estimated deficit power is not identical to the actual value. It is vital to modify the loadshedding amount of each stage to ensure a precise control.

The frequency variation rate of inertia center is recorded maximum at the beginning of disturbance: $\max \left(d f_{C O I} / d \mathrm{t}\right)=-0.8695 \mathrm{~Hz} / \mathrm{s}$. When the frequency reaches the first frequency threshold $49.2 \mathrm{~Hz}$, the $d f_{\mathrm{COI}} / d t$ is measured and $\Delta_{1} \%=4.9 \%$ is calculated by Equation (11). Applying Equation (13), the shedding amount of first step is modified and the actual amount is: $P_{\text {shed } 1}$ ' $=$ $30 \%-4.9 \%=25.1 \%$. Calculate the comprehensive weight of each load node by Equation (11) and distribute the load-shedding amount using Equation (14). The comparison of load shedding amount is listed in Table 1.

The load shedding corrections of each step are $\Delta_{2} \%=$ $1.4 \%, \Delta_{3} \%=0.47 \%$ respectively. The total shedding amount with dynamic correction is $6.77 \%$ less than the base value, which fully demonstrates the effectiveness of the dynamic correction.

In order to validate the effectiveness of the proposed comprehensive weight, three UFLS schemes with dynamic correction are compared. Scheme 1 is the one proposed in this paper with 4 steps; Scheme 2 distributes the shedding amount according to the load characteristics; Scheme 3 sheds load by the load proportion, namely, the lager the load is, the more it sheds. After the activation of first step, the frequency recovery curves of each scheme are shown in Figure 5.

Table 1. The results of dynamic correction.

\begin{tabular}{cccc}
\hline \multirow{2}{*}{ Scheme } & \multicolumn{3}{c}{ Load shedding steps } \\
\cline { 2 - 4 } & $1^{\text {st }}$ step & $2^{\text {nd }}$ step & $3^{\text {rd }}$ step \\
\hline Traditional scheme & $30 \%$ & $30 \%$ & $40 \%$ \\
Dynamic scheme & $25.1 \%$ & $28.6 \%$ & $39.53 \%$ \\
\hline
\end{tabular}

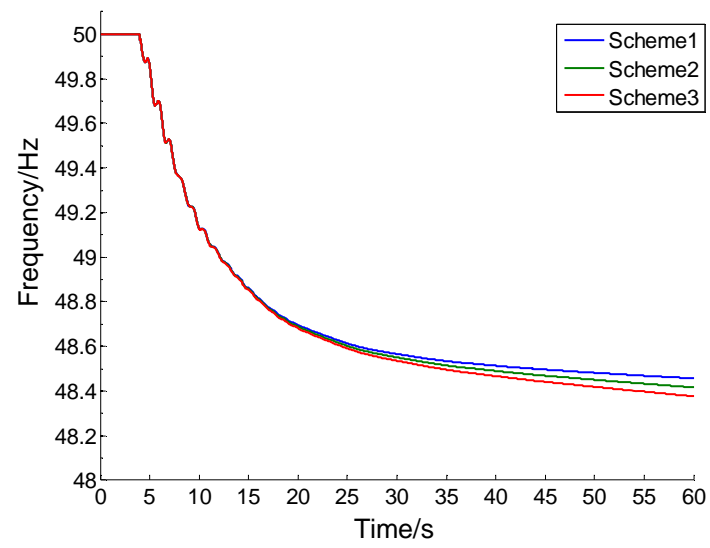

Figure 5. The frequency recovery curves of different underfrequency load shedding schemes after the first step.
It can be seen from Figure 5 that the proposed Scheme 1 can restrain the decrease of frequency quickly due to the fact that enough load-shedding amounts are ensured in the first step. The frequency stabilizes at $48.45668 \mathrm{~Hz}$, better than $48.41676 \mathrm{~Hz}$ of Scheme 2 and $48.37678 \mathrm{~Hz}$ of Scheme 3.

After all the steps activated, the frequency recovery curves are shown in Figure 6 and the shedding amounts are listed in Table 2.

The recovery time of the scheme proposed in this paper is $29.38 \mathrm{~s}$, while Scheme 2 is $32.27 \mathrm{~s}$ and Scheme 3 is $34.96 \mathrm{~s}$. The total load shedding amount of Scheme 1 is 5.0261 p.u, Scheme 2 is 5.0214 p.u and Scheme 3 is 5.0337 p.u. Without considering the load characteristics or other factors, the recovery time and shedding amount of Scheme 3 is unsatisfied. The shedding amount of first step is largest in Scheme 1. However, it is of significance to ensure the load shedding amount in first step to guarantee the frequency recovery. Although, the loads shed of Scheme 1 are more than Scheme 2, the recovery curve rises dramatically, better than that of Scheme 2 and 3. Besides, the recovery time is shortest, which proves that an effective control has been implemented at the key site to meet the demand for control rapidity. Considering the two aspects above, the UFLS scheme with dynamic correction makes full use of the self frequency recovery ability to significantly reduce the total shedding amount compared with the traditional one. Scheme 1 distributes

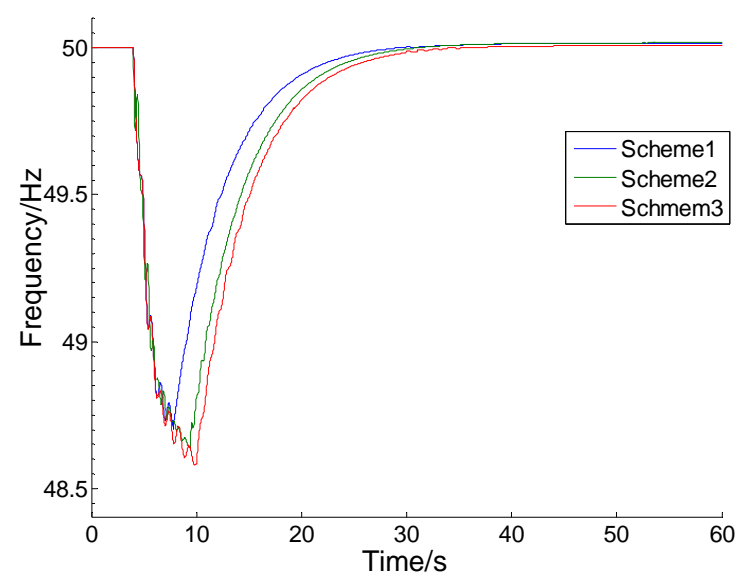

Figure 6. The frequency recovery curves of different under-frequency load shedding schemes.

Table 2. The total amount of shedding load of each scheme.

\begin{tabular}{cccc}
\hline & Scheme 1 & Scheme 2 & Scheme 3 \\
\hline $1^{\text {st }}$ step & 1.3531 & 1.3474 & 1.3489 \\
$2^{\text {nd }}$ step & 1.5419 & 1.5432 & 1.5512 \\
$3^{\text {rd }}$ step & 2.1311 & 2.1308 & 2.1336 \\
total & 5.0261 & 5.0214 & 5.0337 \\
Recovery time & $29.38 \mathrm{~s}$ & $32.27 \mathrm{~s}$ & $34.96 \mathrm{~s}$ \\
\hline
\end{tabular}


loadshedding amount in accordance with the synthetic weigh proposed. Although the shedding amount of scheme1 is not the least, the frequency stabilizes faster at a small cost. Therefore, Scheme 1 is optimal by a comprehensive comparison.

\section{Conclusions}

This paper proposes a new adaptive UFLS scheme base on WAMS, the main research achievements and conclusions are as follows:

1) Combine the UFLS with WAMS and modify the traditional UFLS with adaptive UFLS, effectively shortening the time delay of frequency control.

2) The frequency variation rate of the COI is adopted and the influence of voltage is taken into account to estimate the deficit power.

3) In order to lower the shedding amount, a procedure is given for dynamically adjusting the amount of the shedding steps to adapt to the primary frequency-control response, which reduces the possibility of overcut and unnecessary load losses.

4) A multi-factor comprehensive weight is proposed to distribute the load shedding amount, which facilitates the recovery of frequency as soon as possible.

The proposed method provides a new reference and idea for the online application of frequency-control.

\section{REFERENCES}

[1] Y. S. Xue, X. C. Ren, Q. H. Wu, et al, A Review on Optimization and Coordination of Under Frequency/voltage Load Shedding," Automation of Electric Power Systems,
Vol. 33, No. 9, 2009, pp. 100-107.

[2] V. V. Terzija and J. Koglin, "Adaptive Underfrequency Load Shedding Integrated with A Frequency Estimation Numerical Algorithm," IEEE Proceedings: Generation, Transmission and Distribution, Vol. 149, No. 6, 2002, pp. 713-718.

[3] W. B. Hou, T. Q. Liu and X. Y. Li, "Under-Frequency Load Shedding Based on Comprehensive Weight and Wide Area Measurement System," Power System Protection and Control, Vol. 39, No. 21, 2011, pp. 70-75.

[4] H. Kottickd, "Optimization of Load Shedding System," IEEE Trans Energy Convers, Vol. 8, No. 6, 1993, pp. 593-598.

[5] P. M. Anderson and Mirheydarm, "An Adaptive Method for Setting under Frequency Load Shedding Relays," IEEE Transactions on Power Systems, Vol. 5, 1992, pp. 647-655.

[6] SEETHALEKSHMIK, SINGHSN, SRIVASTAVASC. WAMS Assisted Frequency and Voltage Stability Based Adaptive Load Shedding Scheme, Power\&Energy Society General Meeting Calgary, A, B, 2009, Vol. 1, pp. 1-8.

[7] H. Zhou, Q. Li and T. Lin, "Power System Disturbance and Operation Identification Based on WAMS," Electric Power Automation Equipment, Vol. 31, No. 2, 2011, pp. 7-11.

[8] M. S. Pasand and R. Mihalic, "New Centralized Adaptive under Frequency Load Shedding Algorithms," in Proc. Conf. Power Engineering, 2007 Large Engineering Systems, Oct.10-12. 2007, pp. 44-48.

[9] U. Rudez and R. Mihalic, "Monitoring the First Frequency Derivative to Improve Adaptive Under frequency Load-Shedding Schemes," IEEE Transactions on Power Systems, 2011, Vol. 26, No. 2, pp. 839-846. doi:10.1109/TPWRS.2010.2059715 